\title{
Histologic and Histomorphometric Comparison between Sintered Nanohydroxyapatite and Anorganic Bovine Xenograft in Maxillary Sinus Grafting: A Split-Mouth Randomized Controlled Clinical Trial
}

\author{
Claudio Stacchi, ${ }^{1}$ Teresa Lombardi, ${ }^{2}$ Francesco Oreglia, ${ }^{3}$ \\ Andrea Alberghini Maltoni, ${ }^{4}$ and Tonino Traini ${ }^{5}$ \\ ${ }^{1}$ Department of Medical, Surgical and Health Sciences, University of Trieste, Trieste, Italy \\ ${ }^{2}$ Private Practice, Cassano allo Ionio, Italy \\ ${ }^{3}$ Private Practice, Verona, Italy \\ ${ }^{4}$ Private Practice, Firenze, Italy \\ ${ }^{5}$ Department of Medical, Oral and Biotechnological Sciences, University of Chieti-Pescara, Chieti, Italy \\ Correspondence should be addressed to Claudio Stacchi; claudio@stacchi.it
}

Received 13 January 2017; Accepted 25 May 2017; Published 6 August 2017

Academic Editor: Hassan Maghaireh

Copyright (C) 2017 Claudio Stacchi et al. This is an open access article distributed under the Creative Commons Attribution License, which permits unrestricted use, distribution, and reproduction in any medium, provided the original work is properly cited.

The presence of vital bone after maxillary sinus augmentation is crucial to enhance the quality of bone-implant interface, ensuring predictable long-term results. The aims of this RCT with split-mouth design were the histologic and histomorphometric comparison of two different biomaterials in sinus elevation after 6 months of healing and the evaluation of the clinical outcomes of implants inserted in the augmented areas after 12 months of prosthetic loading. Twenty-eight patients (10 females, 18 males) were treated with bilateral sinus floor elevation with lateral approach. Pure sintered nanohydroxyapatite (NHA) and anorganic bovine bone $(\mathrm{ABB})$ were used as test and active control, respectively. After six months, 52 bone biopsies were harvested from 26 patients, and 107 implants were inserted in the augmented areas. Histomorphometry showed that, in the two groups, vital bone percentages were $34.9 \pm 15 \%(\mathrm{NHA})$ and $38.5 \pm 17 \%(\mathrm{ABB})(p=0.428)$, marrow spaces percentages were $44.5 \pm 18 \%(\mathrm{NHA})$ and $43.5 \pm 23 \%(\mathrm{ABB})$ $(p=0.866)$, and residual graft percentages were $20.6 \pm 13 \%$ (NHA) and $22.3 \pm 12 \%$ (ABB) ( $p=0.638)$. After 6 months of healing, no statistically significant difference was present in histomorphometric outcomes between NHA and ABB groups. Implant survival rate in NHA group after 12 months of loading was 96.4\%, showing no statistically significant differences with ABB group.

\section{Introduction}

Bone resorption and sinus pneumatization are common occurrences in the posterior maxilla after tooth extraction: they may cause both a quantitative reduction and qualitative deterioration of bone, resulting in an inadequate bone volume for dental implant placement [1]. Sinus floor elevation technique had been described more than 35 years ago [2] and extensively studied afterwards, demonstrating high predictability in regenerating bone and allowing for reliable implant supported rehabilitation $[3,4]$. Accurate presurgical planning is a mandatory step: sinus three-dimensional conformation, eventual presence of Underwood septa, and precise localization of the alveolar-antral artery should be assessed and carefully evaluated in order to minimize intraoperative complications and optimize surgical techniques [57].

In general, the quality of osseointegration of dental implants is directly related to the bone-implant contact: in a regenerated tissue, the quantity of newly formed bone is of paramount importance for successful integration of the fixture. Therefore, an adequate biomaterial choice is the first crucial step in bone-implant interface engineering to ensure positive clinical long-term results. 
Autogenous bone had been the first grafting material to be used in sinus floor elevation, being considered as the gold standard option for a long time [8], but its tendency of resorption, its limited availability, and the necessity of a donor site with associated increased morbidity for the patient should be considered as major drawbacks. In the attempt to overcome these limitations, many biomaterials had been proposed and tested, such as allografts, $\beta$-tricalcium phosphate, calcium sulphate, and bone mineral matrix [9-15]. However, anorganic bovine bone (ABB) is probably the most widely investigated bone substitute and, when used in sinus floor elevation, demonstrated satisfactory osteoconductive properties and dimensional stability: from a clinical point of view, implants inserted in ABB-grafted areas showed a high survival rate over time $[16,17]$.

Also, synthetic hydroxyapatites, when used as a bone graft, showed a high degree of biocompatibility and support for cellular activity: they stimulate osteoconduction and are generally slowly replaced by the host bone after implantation [18]. Continuous improvements in synthesis, manufacturing technology, and purification have led to a variety of synthetic HA-based materials with different properties. Among them, synthetic hydroxyapatite with nanoscale porosities seems to favor bone matrix proteins adhesion and to promote differentiation of osteoblast precursor cells [19, 20]. Even if nanocrystalline HA embedded in a highly porous silica gel matrix was already tested as a material for sinus floor elevation [21, 22], a prospective study with a direct comparison between the behavior in the maxillary sinus of pure sintered nanohydroxyapatite (NHA) and ABB in terms of osteoconductive potential was not performed yet.

The aim of this parallel-group, superiority randomized clinical trial (RCT) with split-mouth design was the histologic and histomorphometric comparison for the newly formed tissue after sinus floor elevation with lateral approach performed by using two different grafting materials: NHA as test and $\mathrm{ABB}$ as active control.

An additional aim was the evaluation of the clinical outcomes of dental implants inserted in the augmented areas after 12 months of prosthetic loading. The planned follow-up for this study is five years after prosthetic loading.

\section{Materials and Methods}

2.1. Study Design. The present study was a multicenter randomized controlled clinical trial with a split-mouth design, following CONSORT guidelines, and was conducted in four clinical centers in accordance with the Good Clinical Practice Guidelines (GCPs) and with the recommendations of the Declaration of Helsinki as revised in Fortaleza (2013) for investigations with human subjects. The study protocol had been approved by the relevant ethical committee (Comitato Etico Calabria-Sezione Area Nord) and registered in a public register (NCT03077867).

Patients were thoroughly informed about the protocol, the treatment and its alternatives, the benefits, and the possible risks and signed written informed consent for the participation in the study. This superiority trial tested the null hypothesis of no differences in new bone formation and dental implant survival between NHA bone grafts (test group) and $\mathrm{ABB}$ grafts (active control group) in atrophic maxillae treated with sinus floor elevation with lateral approach.

2.2. Study Population. Eligible participants were adult patients (aged $\geq 18$ years), with severe bilateral maxillary atrophy (crestal height $<3 \mathrm{~mm}$, class V-VI of Cawood and Howell classification [23]) and needing sinus floor elevation to allow for fixed rehabilitation supported by osseointegrated implants, inserted with a staged approach.

Exclusion criteria were

(1) acute myocardial infarction within the past 2 months;

(2) uncontrolled coagulation disorders;

(3) uncontrolled diabetes (HbAlc > 7.5\%);

(4) radiotherapy to the head/neck district within the past 24 months;

(5) immunocompromised patients (HIV infection or chemotherapy within the past 5 years);

(6) present or past treatment with intravenous bisphosphonates;

(7) allergy to bovine collagen;

(8) presence of uncontrolled or untreated periodontal disease;

(9) presence of sinusal pathologies contraindicating sinus floor elevation procedures;

(10) psychological or psychiatric problems;

(11) alcohol or drugs abuse;

(12) patient not fully able to comply with the study protocol;

(13) Schneiderian membrane perforation during surgery.

2.3. Surgical Procedures. Surgical procedures were performed in four centers by experienced operators (CS, TL, FO, and AAM). Patients were draped to guarantee maximum asepsis and perioral skin was disinfected by using iodopovidone $10 \%$ (Betadine, Medifarm, Italy). After performing local anesthesia by using articaine 4\% with epinephrine 1:100.000 (Artin, Omnia, Italy) and raising a full-thickness flap, a window was designed on the lateral wall of the sinus by using ultrasonic instrumentation with the erosion technique (Piezosurgery Touch, Mectron, Italy, and Piezotome, Acteon, France) [24] and the Schneiderian membrane was carefully elevated using ultrasonic inserts and manual curettes. After checking the integrity of the Schneiderian membrane with Valsalva maneuver, the randomization sealed opaque envelope was opened, revealing to the surgeon the grafting material to be used. The biomaterials selected for this study were sintered NHA (Fisiograft Bone Granular, Ghimas, Italy) in the test sites and ABB (Bio-Oss, Geistlich, Switzerland) in the control sites. After the completion of the grafting procedure, the antrostomy was covered by a resorbable bovine collagen membrane (Bio-Gide, Geistlich, Switzerland), fixed with two pins (Micropin, Omnia, Italy), and flaps were sutured with 
Sentineri technique [25] and single stitches using a synthetic monofilament (PTFE, Omnia, Italy).

The contralateral sinus floor augmentation was performed in the same surgical session, with the same surgical protocol, inserting the grafting material not selected in the first intervention.

Patients were prescribed antibiotics for 6 days (amoxicillin $1 \mathrm{~g}$ twice a day or, in allergic patients, clarithromycin $250 \mathrm{mg}$ three times a day) and NSAID (ibuprofen $600 \mathrm{mg}$ ), when needed.

All patients were also advised to sneeze with the mouth open and to avoid nose blowing for two weeks, to prevent unnecessary pressure on the sinus membrane.

Sutures were removed 10 days after surgery. Postsurgical visits were scheduled at monthly intervals to check the course of healing. After six months, bone-core biopsies were collected from the grafted areas using a trephine bur $(3.5 \mathrm{~mm}$ diameter) during the implant bed preparation, and then dental implants (BnxEvo, Ghimas, Italy) were inserted in the harvesting sites. Bone-core specimens were collected with the assistance of surgical templates based upon individual prosthetic requirements. The surgical guides were also used to insert the other programmed implants into the augmented areas: they were left submerged for a four-month healing period, prior to being connected to healing abutments. Finally, implants were restored with screwed metal-ceramic prostheses and patients were followed up for twelve months after loading.

2.4. Histological Analysis. Bone biopsies, left inside the trephine burs, were carefully rinsed for 30-40 seconds with a cold 5\% glucose solution to remove blood maintaining the correct osmolarity $(278 \mathrm{mOsm} / \mathrm{L})$.

The specimens were then placed in Eppendorf tubes with an adequate volume (at least ten times the volume of the specimen) of $10 \%$ formalin solution buffered with phosphate to $\mathrm{pH} 7.2$.

Each specimen was stored in a separate container and labeled. Both patient name and operator ID were noted on a separate sheet to identify the specimens. During the processing phase, both patient name and operator ID were designated by a numerical code.

The specimens were rinsed twice with phosphatebuffered saline and dehydrated with a graded series of alcohol at $4^{\circ} \mathrm{C}$ for seven days. Complete dehydration was then obtained with absolute alcohol immersion for two additional days. Subsequently, the specimens were preinfiltrated in a $50 \%$ resin/alcohol solution (Technovit 7200 VLC, Kulzer, Germany) for ten days and completely embedded in 100\% resin (two changes) using a vacuum chamber for twenty additional days or until the specimens have become transparent.

Finally, specimens were easily removed from the trephine bur using a custom-made plunger (thanks to the shrinkage consequent to dehydration and resin infiltration) and then oriented and polymerized.

After polymerization, the specimens were cut along the longitudinal axis using a high-precision diamond disc at about 50 microns (TT System, TMA2, Italy). The sections were ground under running water to about $30 \pm 10$ microns using a series of polishing discs from 400 to 1200 grits, followed by a final polish with 0.3 -micron alumina in a microgrinding system (TT System, TMA2, Italy).

The prepared sections were stained with Toluidine Blue and Azure II and counterstained with acid fuchsin or doublestained with Toluidine Blue with Pironine G at $1 \%$ and Azure II. The investigation was conducted in a transmitted brightfield microscope (BX 51, Olympus America, USA) and under brightfield/circularly polarized light microscope (Axiolab, Zeiss, Germany) both connected to high-resolution digital cameras (FinePix S2 Pro, Fuji Photo Film, Japan).

Digital photomicrographs were used for histomorphometric analysis, which was performed by a trained and experienced operator (TT). The following parameters were measured: (1) amount of tissue collected with the biopsies over the obtained sections (size of samples); (2) amount of vital bone as absolute value $\left(\mathrm{mm}^{2}\right)$ and as relative value (vital bone area/total sample size $\times 100$ ); (3) marrow space (connective tissue) as absolute value $\left(\mathrm{mm}^{2}\right)$ and as relative value (connective tissue area/total sample size $\times 100$ ); (4) residual grafting material as absolute value $\left(\mathrm{mm}^{2}\right)$ and as relative value (biomaterial area/total sample size $\times 100$ ). A histometric software package with image capturing capabilities (ImagePro Plus 6.0, Media Cybernetics Inc., USA) was used. To ensure accuracy, the software was calibrated for each experimental image using a feature named "Calibration Wizard," which creates a linear remapping of the pixel numbers in millimeters. Intraexaminer variability was controlled by carrying out two measurements for each controlled index. When the difference between the two performed readings exceeded $5 \%$ for the same index, the measurement was repeated.

2.5. Outcomes. This study evaluated the following outcome measures:

(i) Quality of the newly formed tissue: (1) new bone formation (percentage of newly formed bone area to total measured area), (2) residual graft particles (percentage of graft particles area to total measured area), and (3) marrow spaces (percentage of softtissue area to total measured area).

(ii) Implant failure: implant mobility and/or any situation suggesting implant removal.

(iii) Biological and mechanical complications: any complication defined as an unexpected deviation from the normal treatment outcome, both biological (membrane perforation, hemorrhagic events, sinusitis, peri-implantitis, etc.) and mechanical (implant fracture, prosthesis fracture, fixation screw loosening, etc.)

2.6. Sample Size and Statistical Power. Maxillary sinuses were divided into two groups, depending on the grafting material used: a sample size of 24 sinuses per group was needed to detect an effect size of 0.6 on the quantity of newly formed bone (primary outcome), referred to as indicative of a medium effect [26], between the groups (alpha level set at 0.05 and power of $80 \%$ ) (DSS Research, Fort Worth, USA). The effect size is defined as the difference in the given 
TABle 1: Patient characteristics at baseline.

\begin{tabular}{lc}
\hline Males & $18(64.3 \%)$ \\
Females & $10(35.7 \%)$ \\
Mean age (range) & $60.1(39-79)$ \\
Nonsmoker & $18(64.3 \%)$ \\
Light smoker $(<10)$ & $6(21.4 \%)$ \\
Heavy smoker $(\geq 10)$ & $4(14.3 \%)$ \\
Mean residual bone (SD) (range) $(\mathrm{mm})$ & $2.0(0.7)(0.5-3)$ \\
\hline
\end{tabular}

outcome between the groups divided by the within-subjects standard deviations. Each clinical center treated 7 patients with bilateral sinus floor elevation for a total of 56 augmented sinuses to compensate eventual dropouts occurring during the study.

2.7. Randomization. A computer-generated table, distributing right and left sinuses of each patient into two groups (test and control), was prepared using a balanced, randomly permuted block approach (http://www.randomization.com/). The randomization codes were enclosed in numbered, sealed, opaque envelopes which were opened after Schneiderian membrane elevation. Therefore, treatment allocation was concealed to the surgeons in charge of recruiting and treating the patients included in this clinical trial.

2.8. Statistical Analysis. Statistical analysis was performed by means of a computerized statistical package (SigmaStat 3.5, SPSS Inc., Germany). Data were expressed as mean $\pm \mathrm{SD}$ and median (interquartile range), respectively, for parametric and nonparametric values. Items were analyzed with descriptive statistics to assess whether they had a normal distribution; both equal variance and normality tests were used. Considering the two-arm superiority RCT study design, the hypothesis was tested using unpaired $t$-test in case of normally distributed data, while Mann-Whitney $U$ test was performed to compare nonparametric values. A $p$ value $<0.05$ was considered statistically significant.

\section{Results}

3.1. Clinical Results. Twenty-eight patients (aged $60.1 \pm 10.7$ years, range: 39-79 years, 10 females, 18 males) were enrolled, randomized, and treated with bilateral sinus floor elevation with lateral approach. Each clinical center contributed with 7 patients. Eighteen patients were nonsmokers, six light smokers, and four heavy smokers. Preoperative residual bone crest height ranged from 0.5 to $3 \mathrm{~mm}$ (mean height: $2.03 \pm 0.75 \mathrm{~mm}$ ). The main baseline patient characteristics are summarized in Table 1.

Three sinus membrane perforations occurred during elevation in two patients, who dropped out from the study. However, membrane perforations were covered by multiple layers of A-PRF membranes (PRF Duo, Mectron, Italy) and grafting procedures were successfully completed in all of the three cases. No other intraoperative complications were recorded. The healing period following sinus augmentation was uneventful in all patients.

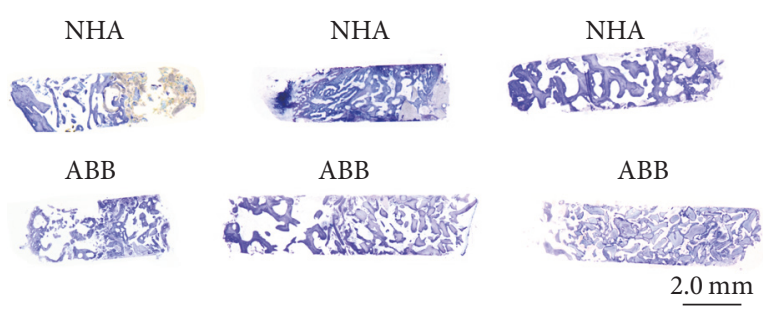

FIGURE 1: Sections of bone cores retrieved after 6 months of healing. NHA: sintered nanohydroxyapatite; ABB: anorganic bovine bone (Toluidine Blue and Azure II; original magnification: 12x).

Six months after sinus floor elevation procedures, 52 bone biopsies were harvested from 26 patients (26 biopsies in test sites, 26 in control sites), and a total of 107 implants were inserted and submerged under the soft tissues (55 implants in test sites, 52 in control sites). After four additional months, at healing abutments connection, three implants in three patients resulted to be not osseointegrated (2.8\% cumulative failure rate): two implants were inserted in test sites (3.6\% failure) and one implant was inserted in a control site ( $1.9 \%$ failure). Difference in implant failure rate between test and control groups was not statistically significant $(p=$ 0.32 , unpaired $t$-test). After an accurate debridement of the implant bed, removed fixtures were immediately replaced with larger diameter implants, which were restored after four additional months of healing. Metal-ceramic screwed prostheses were delivered and, at 12-month follow-up, all implants and prostheses were successfully in function without the occurrence of any biological or mechanical complication.

3.2. Histomorphometric Results. The sections of the harvested biopsies had a mean surface of $9.05 \pm 2.7 \mathrm{~mm}^{2}$ for NHA group and $10.31 \pm 2.9 \mathrm{~mm}^{2}$ for ABB group. The difference between the two groups was not statistically significant $(p=0.116$, unpaired $t$-test). Area of vital bone was $3.29 \pm 2.1 \mathrm{~mm}^{2}$ for NHA group and $4.12 \pm 2.9 \mathrm{~mm}^{2}$ for ABB group. The difference between the two groups was not statistically significant $(p=$ 0.213 , Mann-Whitney $U$ test). Connective tissue area was $3.82 \pm 1.5 \mathrm{~mm}^{2}$ for NHA group and $4.09 \pm 2.3 \mathrm{~mm}^{2}$ for ABB group. The difference between the two groups was not statistically significant ( $p=0.869$, Mann-Whitney $U$ test). The area occupied by residual grafting material was $1.92 \pm$ $1.4 \mathrm{~mm}^{2}$ for NHA group and $2.09 \pm 1.4 \mathrm{~mm}^{2}$ for ABB group. The difference between the two groups was not statistically significant ( $p=0.516$, Mann-Whitney $U$ test). The results are summarized in Table 2 and Figures 1 and 2.

The average percentage of vital bone was $34.9 \pm 15 \%$ for NHA group and $38.5 \pm 17 \%$ for ABB group. The difference between the two groups was not statistically significant $(p=$ 0.428 , unpaired $t$-test). The average percentage of connective tissue was $44.5 \pm 18 \%$ for NHA group and $43.5 \pm 23 \%$ for $\mathrm{ABB}$ group. The difference between the two groups was not statistically significant ( $p=0.866$, unpaired $t$-test). The average percentage of residual grafting material was $20.6 \pm$ $13 \%$ for NHA group and $22.3 \pm 12 \%$ for ABB group. The difference between the two groups was not statistically significant 
TABLE 2: Total area of the analyzed sections $\left[\mathrm{mm}^{2}\right]$.

\begin{tabular}{|c|c|c|c|c|c|c|}
\hline & Samples & Missing & Mean & Std. dev. & Std. error & $\mathrm{CI}$ of mean \\
\hline Total area $\mathrm{ABB}$ & 26 & 0 & 10,319 & 2,923 & 0,573 & 1,180 \\
\hline Total area NHA & 26 & 0 & 9,052 & 2,796 & 0,548 & 1,129 \\
\hline Vital bone $\mathrm{ABB}$ & 26 & 0 & 4,129 & 2,992 & 0,587 & 1,208 \\
\hline Vital bone NHA & 26 & 0 & 3,297 & 2,199 & 0,431 & 0,888 \\
\hline Connective ABB & 26 & 0 & 4,090 & 2,355 & 0,462 & 0,951 \\
\hline Connective NHA & 26 & 0 & 3,828 & 1,510 & 0,296 & 0,610 \\
\hline Biomaterial ABB & 26 & 0 & 2,099 & 1,421 & 0,279 & 0,574 \\
\hline \multirow[t]{2}{*}{ Biomaterial NHA } & 26 & 0 & 1,926 & 1,486 & 0,291 & 0,600 \\
\hline & Range & Max. & Min. & Median & $25 \%$ & $75 \%$ \\
\hline Total area ABB & 12,190 & 18,340 & 6,150 & 9,920 & 8,720 & 11,120 \\
\hline Total area NHA & 10,600 & 14,480 & 3,880 & 8,550 & 7,210 & 11,000 \\
\hline Vital bone ABB & 15,820 & 16,950 & 1,130 & 3,705 & 2,530 & 5,040 \\
\hline Vital bone NHA & 9,700 & 9,960 & 0,260 & 2,750 & 1,840 & 4,630 \\
\hline Connective $\mathrm{ABB}$ & 7,920 & 8,470 & 0,550 & 3,815 & 1,920 & 6,230 \\
\hline Connective NHA & 6,320 & 6,920 & 0,600 & 3,900 & 2,960 & 4,940 \\
\hline Biomaterial ABB & 5,480 & 5,540 & 0,0600 & 1,795 & 1,200 & 3,110 \\
\hline \multirow[t]{2}{*}{ Biomaterial NHA } & 5,380 & 5,530 & 0,150 & 1,530 & 0,810 & 2,770 \\
\hline & Skewness & Kurtosis & K-S dist. & K-S prob. & Sum & Sum of squares \\
\hline Total area $\mathrm{ABB}$ & 0,916 & 0,972 & 0,161 & 0,080 & 268,290 & 2981,976 \\
\hline Total area NHA & 0,260 & $-0,524$ & 0,111 & 0,515 & 235,340 & 2325,652 \\
\hline Vital bone ABB & 3,279 & 13,938 & 0,221 & 0,002 & 107,360 & 667,054 \\
\hline Vital bone NHA & 1,232 & 2,081 & 0,123 & 0,380 & 85,730 & 403,549 \\
\hline Connective $\mathrm{ABB}$ & 0,139 & $-1,039$ & 0,107 & 0,573 & 106,350 & 573,633 \\
\hline Connective NHA & $-0,240$ & $-0,184$ & 0,137 & 0,228 & 99,530 & 437,977 \\
\hline Biomaterial ABB & 0,596 & $-0,208$ & 0,138 & 0,222 & 54,570 & 165,038 \\
\hline Biomaterial NHA & 1,117 & 0,622 & 0,168 & 0,057 & 50,080 & 151,657 \\
\hline
\end{tabular}

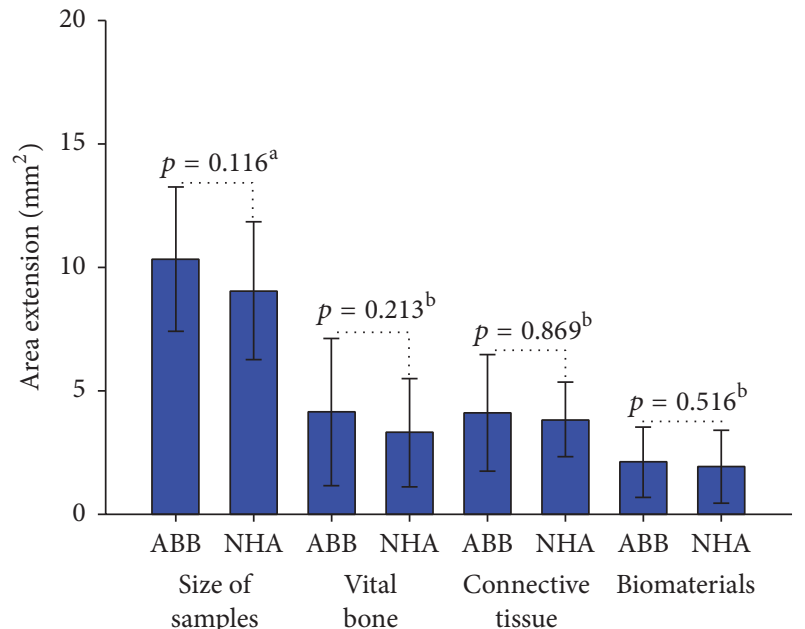

FIGURE 2: Samples area $\left(\mathrm{mm}^{2}\right)$ and surface of vital bone, connective tissue, and biomaterial remnants $\left(\mathrm{mm}^{2}\right)$. NHA: sintered nanohydroxyapatite; ABB: anorganic bovine bone. (a) Unpaired $t$-test; (b): Mann-Whitney $U$ test. Level of significance: $p<0.05$.

( $p=0.638$, unpaired $t$-test). Results are summarized in Table 3 and Figure 3.

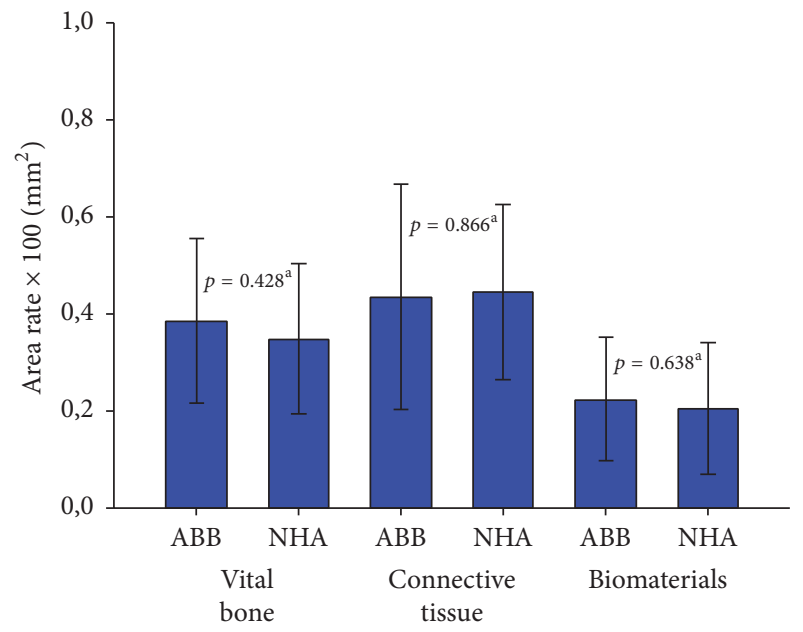

FIGURE 3: Histomorphometric measurements expressed in percentage. NHA: sintered nanohydroxyapatite; ABB: anorganic bovine bone. (a) Unpaired $t$-test. Level of significance: $p<0.05$.

\section{Discussion}

In the clinical practice, the final purpose of bone regeneration is the formation of an adequate quantity of tissue of good 
TABLE 3: Histomorphometric data expressed in percentage [\%].

\begin{tabular}{|c|c|c|c|c|c|c|}
\hline & Samples & Missing & Mean & Std. dev. & Std. error & $\mathrm{CI}$ of mean \\
\hline Vital bone rate $\mathrm{ABB}$ & 26 & 0 & 0,385 & 0,170 & 0,0333 & 0,0685 \\
\hline Vital bone rate NHA & 26 & 0 & 0,349 & 0,155 & 0,0303 & 0,0625 \\
\hline Connective rate $\mathrm{ABB}$ & 26 & 0 & 0,435 & 0,232 & 0,0456 & 0,0939 \\
\hline Connective rate NHA & 26 & 0 & 0,445 & 0,181 & 0,0354 & 0,0729 \\
\hline Biomaterial rate $\mathrm{ABB}$ & 26 & 0 & 0,223 & 0,128 & 0,0252 & 0,0518 \\
\hline \multirow[t]{2}{*}{ Biomaterial rate NHA } & 26 & 0 & 0,206 & 0,135 & 0,0265 & 0,0546 \\
\hline & Range & Max. & Min. & Median & $25 \%$ & $75 \%$ \\
\hline Vital bone rate $\mathrm{ABB}$ & 0,810 & 0,924 & 0,114 & 0,379 & 0,263 & 0,469 \\
\hline Vital bone rate NHA & 0,661 & 0,688 & 0,0270 & 0,339 & 0,279 & 0,465 \\
\hline Connective rate $\mathrm{ABB}$ & 0,942 & 0,990 & 0,0480 & 0,462 & 0,293 & 0,560 \\
\hline Connective rate NHA & 0,709 & 0,786 & 0,0770 & 0,453 & 0,308 & 0,562 \\
\hline Biomaterial rate $\mathrm{ABB}$ & 0,442 & 0,448 & 0,00600 & 0,228 & 0,138 & 0,310 \\
\hline \multirow[t]{2}{*}{ Biomaterial rate NHA } & 0,524 & 0,550 & 0,0260 & 0,161 & 0,130 & 0,309 \\
\hline & Skewness & Kurtosis & K-S dist. & K-S prob. & Sum & Sum of squares \\
\hline Vital bone rate $\mathrm{ABB}$ & 1,256 & 2,846 & 0,131 & 0,288 & 10,015 & 4,576 \\
\hline Vital bone rate NHA & $-0,143$ & $-0,0594$ & 0,126 & 0,345 & 9,079 & 3,768 \\
\hline Connective rate $\mathrm{ABB}$ & 0,381 & 0,0977 & 0,0961 & 0,698 & 11,316 & 6,276 \\
\hline Connective rate NHA & $-0,132$ & $-0,392$ & 0,102 & 0,625 & 11,570 & 5,964 \\
\hline Biomaterial rate $\mathrm{ABB}$ & 0,0123 & $-0,727$ & 0,0758 & 0,858 & 5,800 & 1,706 \\
\hline Biomaterial rate NHA & 0,840 & 0,275 & 0,152 & 0,124 & 5,350 & 1,558 \\
\hline
\end{tabular}

quality, in which to insert dental implants with a good longterm prognosis. Obviously, the biological behavior of the biomaterials is of primary interest. Kirkpatrick et al. underlined the differences between the regenerative processes that have the teleological purpose of "restitutio ad integrum" of the affected tissue and the repair process, which is a structural adaptation to a function task [27]. It was reported that the bone regeneration process with alloplastic, xenograft, and allograft bone substitutes follows three main phases: T1 which is the "time of grafting," with a predominant heterogeneous phase in suspension of blood clot and particles of biomaterials; T2 which is the "time of repairing," with a solid heterogeneous composite phase of biomaterial remnants and newly formed bone; T3 which is the "time of regeneration," with a solid homogeneous phase of newly formed bone without biomaterials remnants. The most common bone substitute biomaterials do not reach the phase $\mathrm{T} 3$ in their clinical use [28]. However, many studies demonstrated that implant osseointegration process can be also obtained and maintained in augmented sinuses where residual graft particles were still present, without a negative influence of biomaterial remnants on peri-implant bone regeneration [29-33].

Hence, even if autologous bone had been traditionally considered as the gold standard to promote new bone regeneration, the choice of alternative biomaterials is now the preferred option in sinus floor elevation for three main reasons: less morbidity, less resorption, and unlimited availability. ABB is the most widespread biomaterial used for sinus grafting and its behavior had been extensively investigated over the years, showing satisfactory long-term results [16, 17].
However, disadvantages of xenografts should also be considered: they include potential risk of prion disease transmission [34] and reaction of the host immune system [35]; in addition, some patients could refuse their use for religious motivations or because they are in contrast with their lifestyle (e.g., vegans and vegetarians). In a recent study, allografts and xenografts elicited the highest refusal rates among the surveyed patients: $15 \%$ of the patients said they would accept a xenograft under no circumstances, while $18 \%$ said they would accept this type of bone graft only as a last resort [36].

The use of synthetic, alloplastic biomaterials could overcome these limitations: they have been studied for years and successfully used in sinus augmentation, but a direct comparison with xenografts, in a split-mouth design, has been reported in the literature by very few and often underpowered trials $[15,37-39]$.

The results of the present RCT showed no statistically significant differences between NHA and ABB groups in terms of new bone formation and survival rate of implants inserted in the augmented areas after 12 months of prosthetic loading: therefore, the null hypothesis tested in this study was accepted.

The histometric comparison after 6 months of healing showed that the osteoconductive potential of NHA is clinically and statistically comparable to $\mathrm{ABB}$, even if it resulted in a slightly lower percentage of vital bone $(34.9 \%$ against $38.5 \%$ ), but showing also a lower percentage of residual grafting material (20.6\% against $22.3 \%)$. Our data are in accordance with other human studies performed by using synthetic hydroxyapatites as sinus grafting material, 

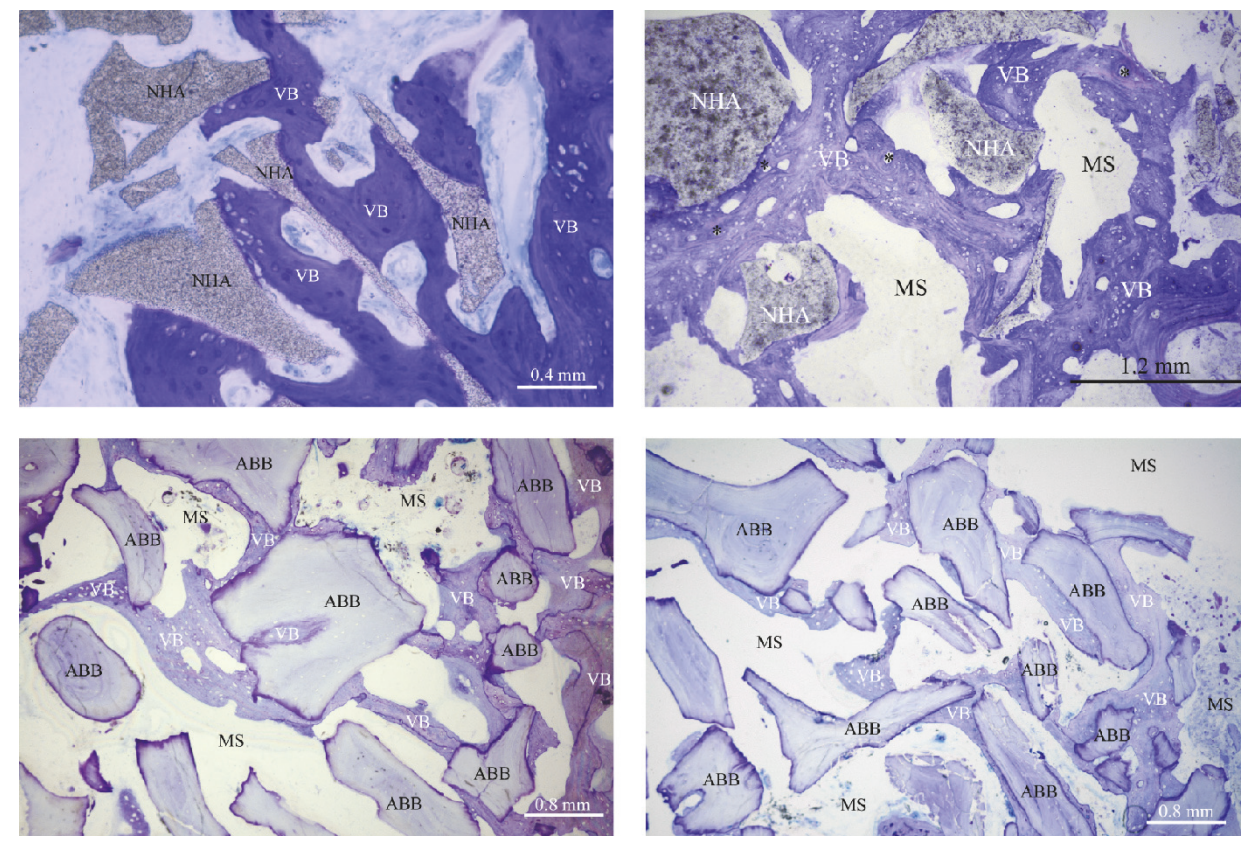

FIGURE 4: Biomaterial particles of both groups appeared to be surrounded and merged by newly formed bone. Several haversian systems (*) were noted in the newly formed bone. NHA: sintered nanohydroxyapatite; ABB: anorganic bovine bone; VB: vital bone; MS: marrow spaces (Toluidine Blue and Azure II; original magnification: 100x).
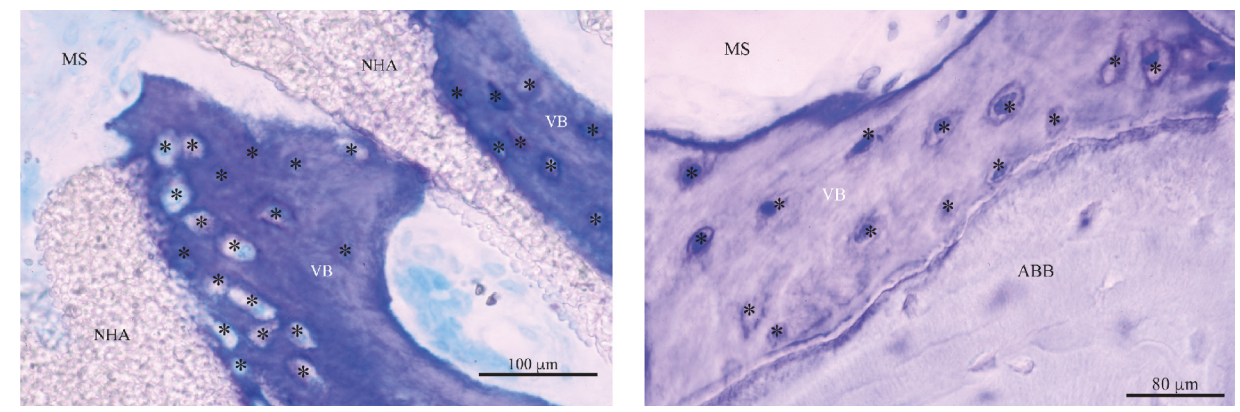

FIGURE 5: The bone around both biomaterials was characterized by the presence of osteocytes $(*)$ embedded inside the mineralized bone matrix. In the test group, osteocytes were generally more numerous near the material surface. NHA: sintered nanohydroxyapatite; ABB: anorganic bovine bone; VB: vital bone; MS: marrow spaces (Toluidine Blue and Azure II; original magnification: 400x).

reporting new bone formation at six months ranging from 32 to $38.5 \%$ [40-42]. These results are also comparable with the histomorphometric outcomes of sinus augmentation performed by using solely autogenous bone (new bone formation at six months ranging from 36.8 to $41 \%$ ) [41, 43-45].

After 6 months of healing, both biomaterials used in the present study showed a good level of "osseointegration," with an adequate extension of bonding surface between host bone and the biomaterial particles. Graft remnants were easily recognizable from the other components of the regenerated tissue and appeared to be merged by bridges of new bone (Figure 4). Furthermore, the bone around the biomaterial particles was characterized by numerous osteocytes embedded into the mineralized bone matrix. These cells, in the test group, were generally more numerous near the biomaterial surface (Figure 5): this feature indicates both a considerable osteointegrative property due to stimulation of the osteoblastic activity and an osteoinductive property of the external surface of the biomaterial (Figure 6). These aspects, according to other studies [46-48], seem particularly related to NHA structure. As described in detail by Kasai et al. [49], cells' proliferation appeared to be stimulated, when in contact with NHA paste, by the activation of epidermal growth factor receptor (EGFR) and its downstream targets serine/threonine protein kinase (AKT) and signal regulated kinases (ERK 1/2). Finally, as expected, both groups showed intense osteoconductive activities (Figure 7).

Implant survival rate in NHA group after 12 months of loading $(96.4 \%)$ showed no statistically significant differences with $\mathrm{ABB}$ group. This outcome is also comparable with 


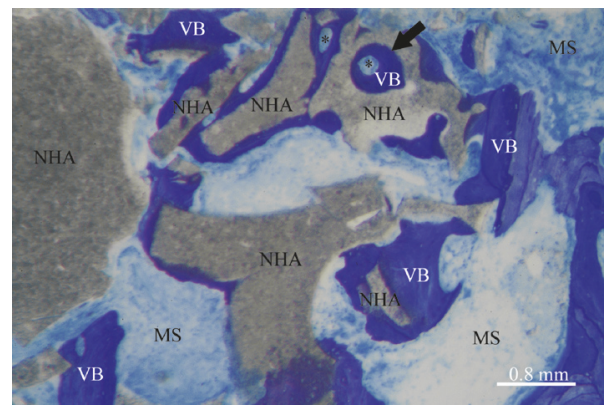

Figure 6: The new bone around some NHA particles presented osteons (*), indicating a relative angiogenetic potential of the material. Vessels' growth was present in pores of adequate dimension inside the biomaterial (black arrow). NHA: sintered nanohydroxyapatite; ABB: anorganic bovine bone; VB: vital bone; MS: marrow spaces (Toluidine Blue and Azure II; original magnification: 100x).
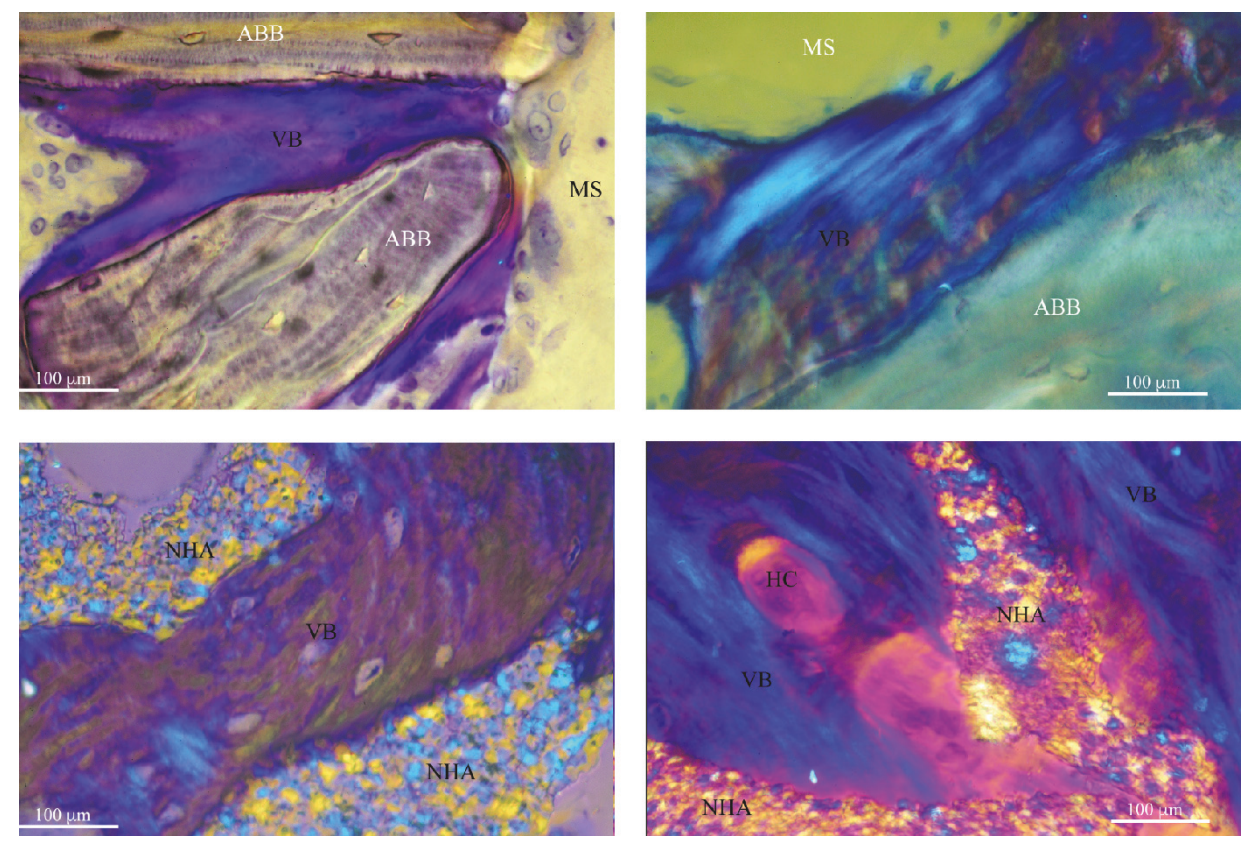

FIGURE 7: Under circularly polarized light, osteoconduction appeared clear with intimate contact between the new bone (VB) and both biomaterials (NHA and ABB). Moreover, the differences in the microstructure (mainly collagen fiber orientation) can be observed. Around some NHA particles, the new bone presented osteons with vessels (HC).

results reported in recent systematic reviews for sinus grafting using solely autogenous bone (97.2-97.4\%) or different bone substitutes $(98.2-98.6 \%)[50,51]$.

Currently, the main limitation of the present study is the relatively short time of follow-up: however, a long-term evaluation of the clinical outcomes in the patients enrolled in this trial had already been coordinated. Furthermore, additional investigations on the biomechanical performances of different bone substitutes would help in determining their appropriate clinical use.

\section{Conclusions}

The findings of the present RCT showed that both NHA and $\mathrm{ABB}$ led to the formation of a regenerated tissue composed of more than $1 / 3$ of vital bone after six months of healing, without any statistically significant difference between test and control groups. NHA could be regarded as a suitable grafting material for clinical cases needing bone augmentation to allow dental implant placement.

The clinical implications of the present study include the possibility of increasing the alternatives for the replacement of bone autografts, which not always represent a possible or convenient option. Sintered NHA couples the benefits of technological advancement with the safety of synthetic biomaterials, preventing the potential risks of xenograft implantation to the patient.

\section{Conflicts of Interest}

The authors declare no conflicts of interest related to this study. 


\section{Acknowledgments}

The authors wish to thank Dr. Rosario Sentineri and Dr. Paolo Cusimano for their valuable help in conducting this study.

\section{References}

[1] A. Monje, F. Monje, R. González-García et al., "Influence of atrophic posterior maxilla ridge height on bone density and microarchitecture," Clinical Implant Dentistry and Related Research, vol. 17, no. 1, pp. 111-119, 2015.

[2] P. J. Boyne and R. A. James, "Grafting of the maxillary sinus floor with autogenous marrow and bone," Journal of Oral Surgery, vol. 38 , no. 8, pp. 613-616, 1980.

[3] M. Del Fabbro, T. Testori, L. Francetti, and R. Weinstein, "Systematic review of survival rates for implants placed in the grafted maxillary sinus," International Journal of Periodontics and Restorative Dentistry, vol. 24, no. 6, pp. 565-577, 2004.

[4] M. Esposito, M. G. Grusovin, J. Rees et al., "Effectiveness of sinus lift procedures for dental implant rehabilitation: a Cochrane systematic review," European Journal of Oral Implantology, vol. 3, no. 1, pp. 7-26, 2010.

[5] C. Stacchi, F. Andolsek, F. Berton, G. Perinetti, C. O. Navarra, and R. Di Lenarda, "Intraoperative complications during sinus floor elevation with lateral approach: a systematic review," International Journal of Oral and Maxillofacial Implants, vol. 32, no. 3, pp. e107-e118, 2017.

[6] P. Maridati, E. Stoffella, S. Speroni, M. Cicciù, and C. Maiorana, "Alveolar antral artery isolation during sinus lift procedure with the double window technique," Open Dentistry Journal, vol. 8, no. 8, pp. 95-103, 2014.

[7] D. Rancitelli, A. E. Borgonovo, M. Cicciù et al., "Maxillary sinus septa and anatomic correlation with the Schneiderian membrane," Journal of Craniofacial Surgery, vol. 26, no. 4, pp. 1394-1398, 2015.

[8] S. A. Danesh-Sani, S. P. Engebretson, and M. N. Janal, "Histomorphometric results of different grafting materials and effect of healing time on bone maturation after sinus floor augmentation: a systematic review and meta-analysis," Journal of Periodontal Research, vol. 52, no. 3, pp. 301-312, 2017.

[9] C. Stacchi, G. Orsini, D. Di Iorio, L. Breschi, and R. Di Lenarda, "Clinical, histologic, and histomorphometric analyses of regenerated bone in maxillary sinus augmentation using fresh frozen human bone allografts," Journal of Periodontology, vol. 79, no. 9, pp. 1789-1796, 2008.

[10] J. Wu, B. Li, and X. Lin, "Histological outcomes of sinus augmentation for dental implants with calcium phosphate or deproteinized bovine bone: a systematic review and metaanalysis," International Journal of Oral and Maxillofacial Surgery, vol. 45, no. 11, pp. 1471-1477, 2016.

[11] M. Pettinicchio, R. Sammons, S. Caputi, A. Piattelli, and T. Traini, "Bone regeneration in sinus augmentation procedures with calcium sulphate. Microstructure and microanaytical investigations," Australian Dental Journal, vol. 57, no. 2, pp. 200206, 2012.

[12] M. Nevins, F. Heinemann, U. W. Janke et al., "Equine-derived bone mineral matrix for maxillary sinus floor augmentation: a clinical, radiographic, histologic, and histomorphometric case series," International Journal of Periodontics and Restorative Dentistry, vol. 33, no. 4, pp. 483-489, 2013.
[13] G. Pinchasov and G. Juodzbalys, "Graft-free sinus augmentation procedure: a literature review," Journal of Oral and Maxillofacial Research, vol. 5, no. 1, 2014.

[14] L. Laino, G. Troiano, G. Giannatempo et al., "Sinus lift augmentation by using calcium sulphate. A retrospective 12 months radiographic evaluation over 25 treated Italian patients," Open Dentistry Journal, vol. 9, pp. 414-419, 2015.

[15] C. K. Dursun, E. Dursun, K. Eratalay et al., "Effect of porous titanium granules on bone regeneration and primary stability in maxillary sinus: A human clinical, histomorphometric, and microcomputed tomography analyses," Journal of Craniofacial Surgery, vol. 27, no. 2, pp. 391-397, 2016.

[16] C. E. A. Ferreira, A. B. Novaes Jr., V. I. Haraszthy, M. Bittencourt, C. B. Martinelli, and S. M. Luczyszyn, "A clinical study of 406 sinus augmentations with $100 \%$ anorganic bovine bone," Journal of Periodontology, vol. 80, no. 12, pp. 1920-1927, 2009.

[17] D. Z. Lee, S. T. Chen, and I. B. Darby, "Maxillary sinus floor elevation and grafting with deproteinized bovine bone mineral: a clinical and histomorphometric study," Clinical Oral Implants Research, vol. 23, no. 8, pp. 918-924, 2012.

[18] R. E. Holmes, R. W. Bucholz, and V. Mooney, "Porous hydroxyapatite as a bone-graft substitute in metaphyseal defects. A histometric study," Journal of Bone and Joint Surgery, vol. 68, no. 6, pp. 904-911, 1986.

[19] T. Gerber, G. Holzhüter, W. Götz, V. Bienengräber, K.-O. Henkel, and E. Rumpel, "Nanostructuring of biomaterials A pathway to bone grafting substitute," European Journal of Trauma, vol. 32, no. 2, pp. 132-140, 2006.

[20] W. Götz, T. Gerber, B. Michel, S. Lossdörfer, K.-O. Henkel, and F. Heinemann, "Immunohistochemical characterization of nanocrystalline hydroxyapatite silica gel (NanoBone $\left.{ }^{\circledR}\right)$ osteogenesis: A study on biopsies from human jaws," Clinical Oral Implants Research, vol. 19, no. 10, pp. 1016-1026, 2008.

[21] L. Canullo, C. Dellavia, and F. Heinemann, "Maxillary sinus floor augmentation using a nano-crystalline hydroxyapatite silica gel: case series and 3-month preliminary histological results," Annals of Anatomy, vol. 194, no. 2, pp. 174-178, 2012.

[22] D. D. Bosshardt, M. M. Bornstein, J.-P. Carrel, D. Buser, and J.-P. Bernard, "Maxillary sinus grafting with a synthetic, nanocrystalline hydroxyapatite-silica gel in humans: histologic and histomorphometric results," The International Journal of Periodontics \& Restorative Dentistry, vol. 34, no. 2, pp. 259-267, 2014.

[23] J. I. Cawood and R. A. Howell, "A classification of the edentulous jaws," International Journal of Oral and Maxillofacial Surgery, vol. 17, no. 4, pp. 232-236, 1988.

[24] C. Stacchi, T. Vercellotti, A. Toschetti, S. Speroni, S. Salgarello, and R. Di Lenarda, "Intraoperative complications during sinus floor elevation using two different ultrasonic approaches: A two-center, randomized, controlled clinical trial," Clinical Implant Dentistry and Related Research, vol. 17, supplement 1, pp. e117-e125, 2015.

[25] R. Sentineri, T. Lombardi, F. Berton, and C. Stacchi, "LaurellGottlow suture modified by sentineri for tight closure of a wound with a single line of sutures," British Journal of Oral and Maxillofacial Surgery, vol. 54, no. 1, pp. e18-e19, 2016.

[26] J. Cohen, “A power primer," Psychological Bulletin, vol. 112, no. 1, pp. 155-159, 1992.

[27] C. J. Kirkpatrick, V. Krump-Konvalinkova, R. E. Unger, F. Bittinger, M. Otto, and K. Peters, "Tissue response and biomaterial integration: the efficacy of in vitro methods," Biomolecular Engineering, vol. 19, no. 2-6, pp. 211-217, 2002. 
[28] T. Traini, A. Piatelli, and S. Caputi, "Regeneration of human bone using different bone substitute biomaterials," Clinical Implant Dentistry and Related Research, vol. 17, no. 1, pp. 150162, 2015.

[29] P. Valentini, D. Abensur, D. Densari, J. N. Graziani, and C. Hämmerle, "Histological evaluation of Bio-Oss in a 2-stage sinus floor elevation and implantation procedure. A human case report," Clinical Oral Implants Research, vol. 9, no. 1, pp. 59-64, 1998.

[30] J. L. Rosenlicht and D. P. Tarnow, "Human histologic evidence of integration of functionally loaded hydroxyapatite-coated implants placed simultaneously with sinus augmentation: a case report 2 1/2 years postplacement," Journal of Oral Implantology, vol. 25, no. 1, pp. 7-10, 1999.

[31] A. Scarano, G. Pecora, M. Piattelli, and A. Piattelli, "Osseointegration in a sinus augmented with bovine porous bone mineral: histological results in an implant retrieved 4 years after insertion. A case report," Journal of Periodontology, vol. 75, no. 8, pp. 1161-1166, 2004.

[32] G. Iezzi, A. Scarano, C. Mangano, B. Cirotti, and A. Piattelli, "Histologic results from a human implant retrieved due to fracture 5 years after insertion in a sinus augmented with anorganic bovine bone," Journal of Periodontology, vol. 79, no. 1, pp. 192-198, 2008.

[33] G. Menicucci, F. Mussano, G. Schierano et al., "Healing properties of implants inserted concomitantly with anorganic bovine bone. A histomorphometric human study," Australian Dental Journal, vol. 58, no. 1, pp. 57-66, 2013.

[34] Y. Kim, H. Nowzari, and S. K. Rich, "Risk of prion disease transmission through bovine-derived bone substitutes: a systematic review," Clinical Implant Dentistry and Related Research, vol. 15, no. 5, pp. 645-653, 2013.

[35] S. R. Bannister and C. A. Powell, "Foreign body reaction to anorganic bovine bone and autogenous bone with platelet-rich plasma in guided bone regeneration," Journal of Periodontology, vol. 79, no. 6, pp. 1116-1120, 2008.

[36] R. F. Fernández, C. Bucchi, P. Navarro, V. Beltrán, and E. Borie, "Bone grafts utilized in dentistry: an analysis of patients' preferences," BMC Medical Ethics, vol. 16, no. 1, 77 pages, 2015.

[37] G. L. de Lange, J. R. Overman, E. Farré-Guasch et al., "A histomorphometric and micro-computed tomography study of bone regeneration in the maxillary sinus comparing biphasic calcium phosphate and deproteinized cancellous bovine bone in a human split-mouth model," Oral Surgery, Oral Medicine, Oral Pathology and Oral Radiology, vol. 117, no. 1, pp. 8-22, 2014.

[38] A. Shirmohammadi, L. Roshangar, M. T. Chitsazi, R. Pourabbas, M. Faramarzie, and N. Rahmanpour, "Comparative study on the efficacy of anorganic bovine bone (Bio-Oss) and nanocrystalline hydroxyapatite (Ostim) in maxillary sinus floor augmentation," International Scholarly Research Notices, vol. 2014, Article ID 967091, 7 pages, 2014.

[39] J. Lorenz, A. Kubesch, T. Korzinskas et al., “TRAP-positive multinucleated giant cells are foreign body giant cells rather than osteoclasts: results from a split-mouth study in humans," Journal of Oral Implantology, vol. 41, no. 6, pp. e257-e266, 2015.

[40] C. Mangano, A. Scarano, G. Iezzi et al., "Maxillary sinus augmentation using an engineered porous hydroxyapatite: a clinical, histological, and transmission electron microscopy study in man," Journal of Oral Implantology, vol. 32, no. 3, pp. 122-131, 2006.

[41] A. Scarano, M. Degidi, G. Iezzi et al., "Maxillary sinus augmentation with different biomaterials: a comparative histologic and histomorphometric study in man," Implant Dentistry, vol. 15, no. 2, pp. 197-207, 2006.

[42] S. M. Belouka and F. P. Strietzel, "Sinus floor elevation and augmentation using synthetic nanocrystalline and nanoporous hydroxyapatite bone substitute materials: preliminary histologic results," International Journal of Oral and Maxillofacial Implants, vol. 31, no. 6, pp. 1281-1291, 2016.

[43] E. S. Tadjoedin, G. L. De Lange, D. M. Lyaruu, L. Kuiper, and E. H. Burger, "High concentrations of bioactive glass material (BioGran) vs. autogenous bone for sinus floor elevation," Clinical Oral Implants Research, vol. 13, no. 4, pp. 428-436, 2002.

[44] S. A. Danesh-Sani, S. S. Wallace, A. Movahed et al., "Maxillary sinus grafting with biphasic bone ceramic or autogenous bone: clinical, histologic, and histomorphometric results from a randomized controlled clinical trial," Implant Dentistry, vol. 25, no. 5, pp. 588-593, 2016.

[45] H. D. Netto, M. D. Miranda Chaves, B. Aatrstrup, R. Guerra, and S. Olate, "Bone formation in maxillary sinus lift using autogenous bone graft at 2 and 6 months," International Journal of Morphology, vol. 34, no. 3, pp. 1069-1075, 2016.

[46] M. Yamada, T. Ueno, N. Tsukimura et al., "Bone integration capability of nanopolymorphic crystalline hydroxyapatite coated on titanium implants," International Journal of Nanomedicine, vol. 7, pp. 859-873, 2012.

[47] V. P. Singh, D. G. Nayak, and D. Shah, “Clinical and radiographic evaluation of nano-crystalline hydroxyapatite bone graft (Sybograft) in combination with bioresorbable collagen membrane (Periocol) in periodontal intrabony defects," Dental Research Journal (Isfahan), vol. 9, no. 1, pp. 60-67, 2012.

[48] Y. Qu, P. Wang, Y. Man, Y. Li, Y. Zuo, and J. Li, "Preliminary biocompatible evaluation of nano-hydroxyapatite/polyamide 66 composite porous membrane," International Journal of Nanomedicine, vol. 5, no. 1, pp. 429-435, 2010.

[49] A. Kasai, B. Willershausen, C. Reichert, B. Röhrig, R. Smeets, and M. Schmidt, "Ability of nanocrystalline hydroxyapatite paste to promote human ligament cell proliferation," Journal of Oral Science, vol. 50, no. 3, pp. 279-285, 2008.

[50] D. Rickert, J. J. R. H. Slater, H. J. A. Meijer, A. Vissink, and G. M. Raghoebar, "Maxillary sinus lift with solely autogenous bone compared to a combination of autogenous bone and growth factors or (solely) bone substitutes. A systematic review," International Journal of Oral and Maxillofacial Surgery, vol. 41, no. 2, pp. 160-167, 2012.

[51] B. Al-Nawas and E. Schiegnitz, "Augmentation procedures using bone substitute materials or autogenous bone-a systematic review and meta-analysis," European Journal of Oral Implantology, vol. 7, no. 2, pp. S219-S234, 2014. 


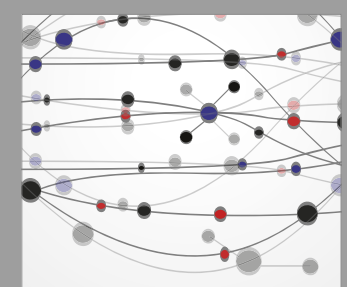

The Scientific World Journal
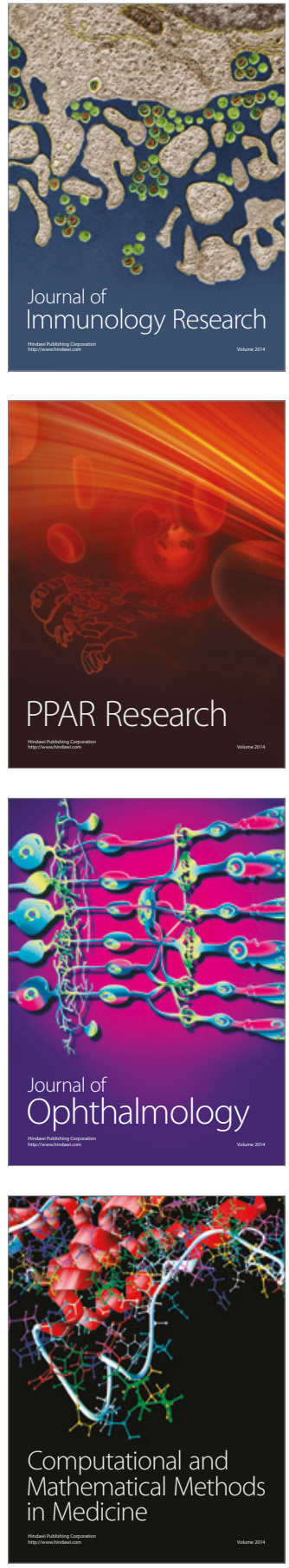

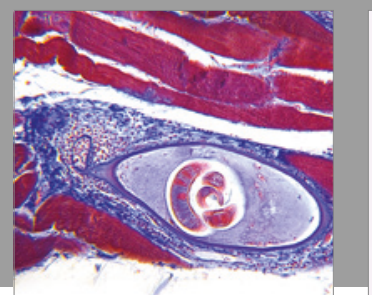

Gastroenterology Research and Practice
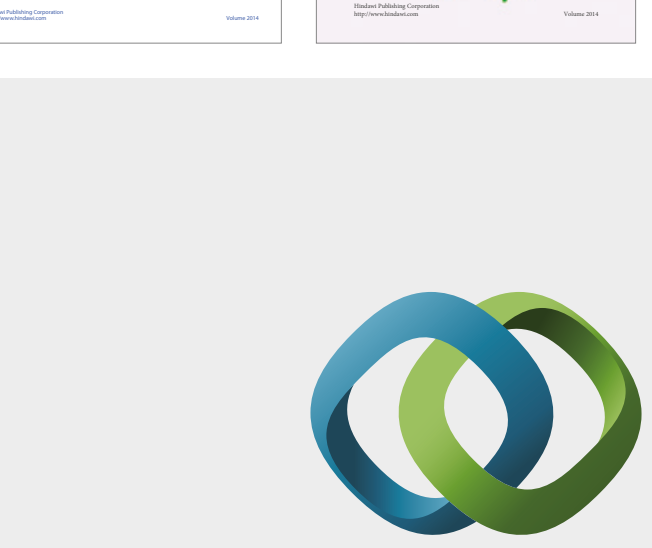

\section{Hindawi}

Submit your manuscripts at

https://www.hindawi.com
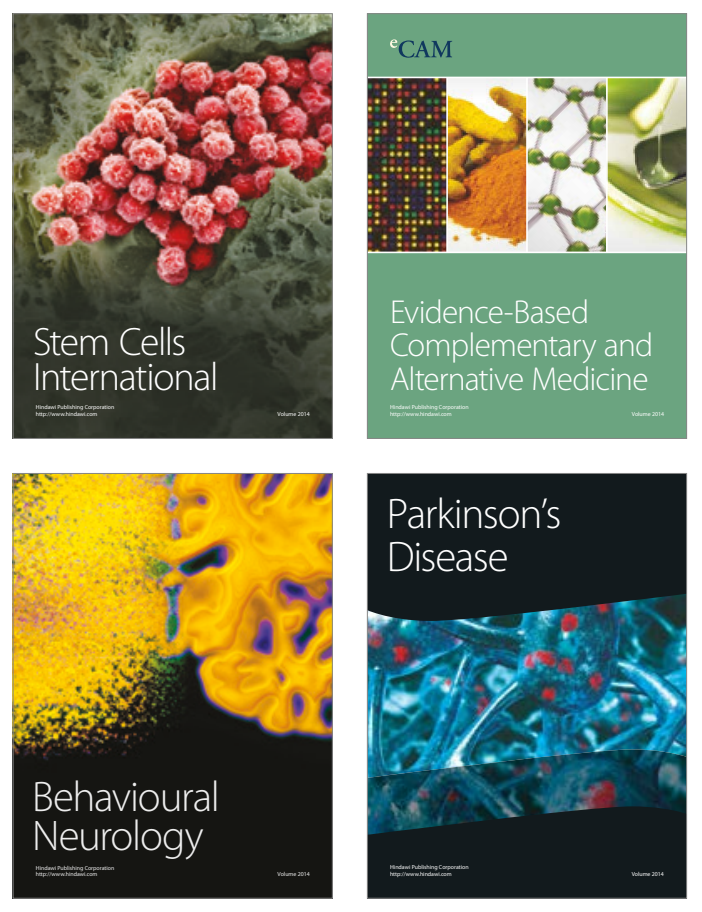
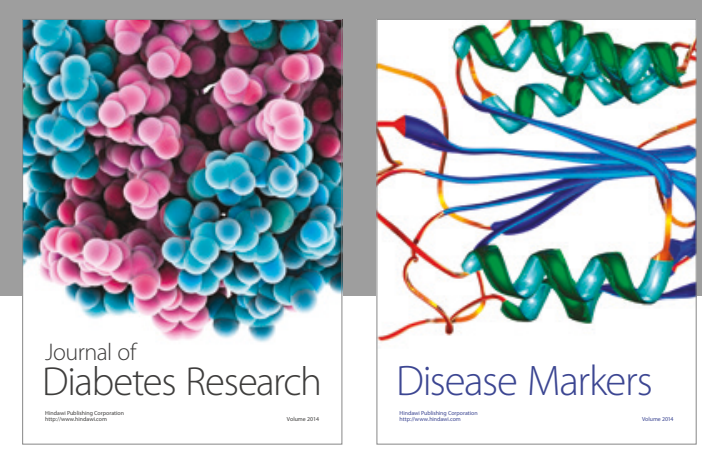

Disease Markers
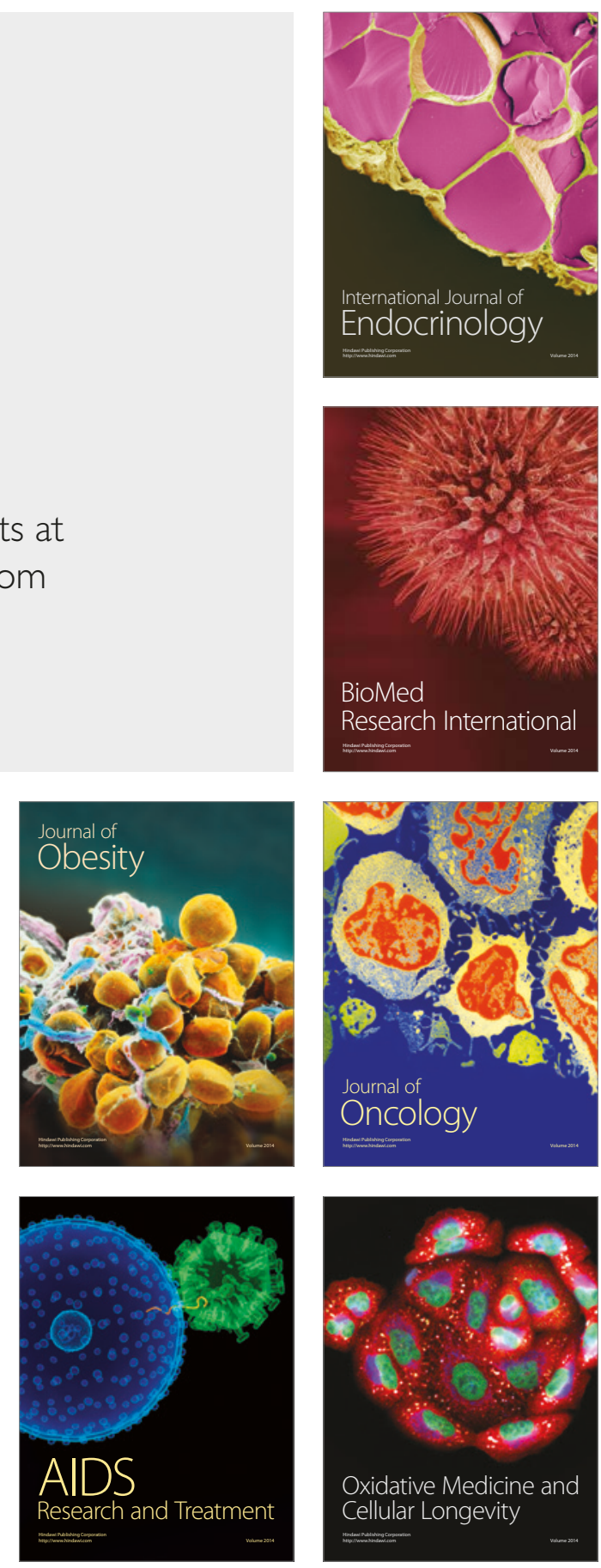